\title{
Wie kann Reden heilen? Zur Sprache in der Psychotherapie
}

\author{
Christopher Marx $\cdot$ Cord Benecke $\cdot$ Antje Gumz
}

Online publiziert: 3. Februar 2020

(C) Der/die Autor(en) 2020

Zusammenfassung Psychotherapie gilt traditionell als „talking cure“, als Heilverfahren also, das wesentlich über Sprache operiert. Unklar ist allerdings, was diese Zuschreibung konkret bedeutet, insofern die therapeutische Wirkung von Sprache unterschiedlich expliziert wurde. Vor diesem Hintergrund rekonstruiert diese Studie zentrale Ansätze, die die ,talking cure“ in Termini von 1. Katharsis, 2. Symbolisierung, 3. Metaphern, 4. verbalen Interventionen und 5. Narrativen beschreiben, und diskutiert diese im Hinblick auf Befunde der Psychotherapieforschung. Im Resultat ergibt sich ein komplexes Bild disparater theoretischer Zugriffe, die in der Grundannahme konvergieren, das therapeutische Potenzial von Sprache in ihrer Möglichkeit zur Explikation beziehungsweise zur Modifikation pathologisch-maladaptiver Erlebens- und Erfahrungskonstellationen zu sehen. Die empirische Befundlage bezüglich dieser Ansätze ist heterogen: Die meisten Typen verbaler Aktivität zeigen unklare Assoziationen mit dem Behandlungserfolg. Konsistenter sind Zusammenhänge mit Prozessvariablen, wobei spezifische Prozesse von unterschiedlichen verbalen Aktivitäten evoziert werden können (zum Beispiel Verstehen durch Übertragungsdeutungen, Selbstoffenbarungen, Metaphern oder Narrative). Weiterhin wurde gezeigt, dass verbale Aktivität auch negative Effekte haben kann. Insgesamt legen diese Befunde eine integrative Sicht auf die Sprache im Sinne eines Sets sprachinduzierter veränderungsrelevanter Prozesse nahe, deren heilsame Wirkung im Kontext

\footnotetext{
Dipl.-Psych. C. Marx $(\varangle) \cdot$ Prof. Dr. med. A. Gumz

Fachbereich für Psychosomatik und Psychotherapie, Psychologische Hochschule Berlin, Am Köllnischen Park 2, 10179 Berlin, Deutschland

E-Mail: praxis-marx@posteo.de

Prof. Dr. phil. Dipl.-Psych. C. Benecke

Institut für Psychologie, Universität Kassel, Holländische Str. 36, 34127 Kassel, Deutschland

Prof. Dr. med. A. Gumz

Institut und Poliklinik für Psychosomatische Medizin und Psychotherapie, Universitäts-Klinikum Hamburg-Eppendorf, Martinistr. 52, 20246 Hamburg, Deutschland
} 
eines umfassenden Beziehungsgeschehens zu betrachten ist und dabei komplexen Randbedingungen unterliegt, die zu präzisieren und systematisieren ein wichtiges Desiderat der Psychotherapieforschung darstellt.

\section{How can talking cure? On language in psychotherapy}

Abstract Psychotherapy is traditionally considered as a talking cure, i.e. a healing method that operates through language; however, the implications of this view are unclear as the therapeutic effects of verbal processes have been spelled out in various ways. Against this background the present study discusses central approaches to the talking cure in terms of 1) catharsis, 2) symbolization, 3) metaphors, 4) verbal interventions and 5) narratives with reference to findings of psychotherapy research. As a result, a complex pattern of disparate theoretical approaches arises which converge in the basic assumption that the therapeutic potential of language is due to its ability to explicate or modify pathological or maladaptive experiential constellations. Empirical findings regarding these approaches are heterogeneous: Most types of verbal activity show equivocal associations with therapy outcome. More consistent findings are reported regarding associations with process variables, which imply that specific processes can be effectuated by different types of verbal activity (e.g. understanding by transference interpretations, self-disclosure, metaphors, or narratives). Moreover, it was demonstrated that most verbal activities can have detrimental effects. Altogether, empirical findings suggest an integrative view on the therapeutic effects of language, i.e., that language contributes to effectuate therapeutic change due to a set of basic processes that need to be considered within the framework of relational interaction and which depend on complex boundary conditions that need to be clarified and systematized in subsequent psychotherapy research.

\section{Psychotherapie als ,talking cure“}

Psychotherapie gilt traditionell als ,talking cure“ (Breuer und Freud 1895, S. 50), als Heilverfahren also, das über einen „Austausch von Worten“ (Freud 1916, S. 43) operiert. Zwar hat sich diese Perspektive differenziert, etwa mit Blick auf den Befund, dass therapeutische Interaktionen maßgeblich von nonverbalen Faktoren geprägt werden (Benecke et al. 2005), beziehungsweise dass therapeutische Veränderungsprozesse als Resultat einer Vielzahl von Faktoren gelten müssen, die nicht umstandslos als sprachliche Faktoren klassifizierbar sind (Tschacher et al. 2014). Gleichwohl wird die Sprache nach wie vor als Leitmedium psychotherapeutischer Praxis betrachtet (Marx et al. 2017), die in diesem Sinne auch in der zeitgenössischen Forschung noch als „talk treatment“ (Wampold 2007, S. 863) oder „talk therapy“ (Woolfolk 2015, S. 2) charakterisiert wird. Fraglich bleibt, was diese Zuschreibung impliziert: Wie kann Reden (,talking“) heilen (,cure“)? Diese Frage wurde unterschiedlich beantwortet, was eine integrative Spezifikation der therapeutischen Effekte sprachlicher Prozesse erschwert.

Ziel der vorliegenden Studie ist es, sich im Rahmen eines narrativen Reviews Aufschluss über die therapeutische Wirkung von Sprache - im Sinne des mani- 
festen Aspekts gesprochener Sprache - zu verschaffen, indem (a) zentrale Ansätze der ,talking cure“ rekonstruiert und diese (b) im Licht der Psychotherapieforschung diskutiert werden. Im Fokus stehen fünf Zugriffsweisen auf die ,talking cure“, die heilsame Effekte sprachlicher Prozesse in Termini von 1. Katharsis, 2. Symbolisierung, 3. Metaphern, 4. verbalen Interventionen und 5. Narrativen beschreiben.

\section{Katharsis}

Der erste Ansatz beschreibt die ,talking cure“ in Termini kathartischer Prozesse. Bezugspunkt ist dabei die Katharsislehre der Antike, in der Katharsis zunächst unspezifisch einen Prozess der Reinigung bezeichnete. Leitend für die weitere Rezeption war die Tragödientheorie der aristotelischen Poetik (1997), in der Katharsis eine Reinigung von Affekten impliziert. Dieses Verständnis war für die psychotherapeutische Aneignung des Konzepts prägend, erstmals in den Studien über Hysterie von Breuer und Freud (1895), die die ,talking cure“ (S. 50) im Sinne der ,,kathartischen Methode“" zur Behandlung „hysterischer Phänomene“ (S. 23) explizieren. Ausgangspunkt ist die Annahme, dass Hysterien durch psychische Traumata verursacht werden, auf die nicht angemessen reagiert werden konnte, woraufhin die traumatische Erinnerung verdrängt, der mit ihr assoziierte Affektbetrag aber als ,eingeklemmte[r] Affekt“ (Breuer und Freud 1895, S. 40) erhalten bleibt und durch „Konversion“ in „körperliche Dauersymptome“ (S. 105) umgesetzt wird. Die ,talking cure“ wird dann als zweistufiger Prozess konstruiert: Im ersten Schritt geht es um eine verbale Reproduktion des traumatischen Ereignisses, das ,in möglichst ausführlicher Wei-

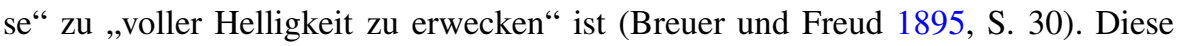
Reproduktion allein reicht aber nicht aus: Im zweiten Schritt geht es darum, ,den begleitenden Affekt wachzurufen“, der vorher nicht abreagiert werden konnte, wobei entscheidend ist, den Affekt zu verbalisieren, ihm „Ablauf durch die Rede [zu] gestatte[n]“ (Breuer und Freud 1895, S. 40), was zur kathartischen Reinigung und letztlich zur Auflösung der hysterischen Symptomatik führt.

In der Nachfolge der Studien wurde das Katharsiskonzept modifiziert. Dabei zeichnet sich eine Entkoppelung des Konzepts vom Störungsbild der Hysterie ab: Kathartische Reinigung gilt mittlerweile als basaler therapeutischer Prozess und mithin als allgemeiner Wirkfaktor der Psychotherapie (Tschacher et al. 2014). Weiterhin legen Forschungsbefunde Differenzierungen bezüglich der mit der Katharsistheorie assoziierten sprachlichen Aktivitäten nahe, was im Folgenden mit Blick auf den Aspekt der Selbstoffenbarung (,disclosure“) sowie der Emotionsverbalisierung gezeigt wird.

Zunächst zur Selbstoffenbarung. In den Studien sieht die „talking cure“ im ersten Schritt vor, bislang verdrängte traumatische Erfahrungen zu rekapitulieren. Im Kern geht es also um die Hypothese, dass das Aussprechen von bislang Nicht-Ausgesprochenem hilfreich ist. Die Effekte solcher Selbstoffenbarungsprozesse wurden - abgelöst vom Kontext der Studien - in der Forschung kontrovers diskutiert (Farber 2003). Ausgangspunkt war der Befund, dass Selbstoffenbarungsprozesse einen wesentlichen Anteil dessen ausmachen, was Patienten in Therapien sagen (Stiles 1995). Parallel zeigte sich aber auch, dass Patienten in Therapien vieles verschwei- 
gen (Farber 2003). Der Effekt solcher „,(Non-)disclosure“-Prozesse ist umstritten: Selbstoffenbarungen sind - erstens - mit dem Therapie-Outcome (also dem Behandlungserfolg, operationalisiert zum Beispiel als Reduktion belastender Symptome) in der Regel nicht oder nur schwach assoziiert (Farber 2003). Gleichzeitig zeigen sich zweitens - positive Assoziationen mit Prozessvariablen: Selbstoffenbarungsprozesse gehen zum Beispiel mit Gefühlen der Erleichterung, höheren Leveln von ,experiencing“, Verstehensprozessen sowie einer besseren therapeutischen Beziehung einher (Farber 2003). Verkompliziert wird die Befundlage - drittens - dadurch, dass verschiedene Studien auch negative Effekte berichten, insofern „Disclosure“-Prozesse mit erhöhtem Stresserleben einhergehen (Stiles 1995) - was nahelegt, dass Selbstoffenbarungsprozesse für den Patienten mit Risiken verbunden sind. Diese Position wurde insbesondere von Kelly (1998) vertreten, die argumentierte, dass die Zurückhaltung persönlicher Details in der Therapie vorteilhaft sein kann. In der Forschung fand dieses Argument eher kritische Resonanz. Favorisiert wurde eine Deutungslinie, die zum Beispiel Stiles (1995) in seinem „fever model of self-disclosure“ formulierte. In diesem Modell gelten Selbstoffenbarungsprozesse als Mittel zum Abbau subjektiver Belastung: Selbstoffenbarung verhalte sich zu Belastungserleben wie Fieber zur körperlichen Infektion, ,both an indicator of some underlying disturbance and part of a restorative process“ (Stiles 1995, S. 82). In diesem Sinne wären Selbstoffenbarungen als Teilaspekt eines homöostatischen Systems zu betrachten: „Distress helps promote Disclosure and Disclosure helps relieve distress and hence reduce the need to Disclose“" (Stiles 1995, S. 83). Diese Sichtweise könnte erklären, weshalb hohe Level von Selbstoffenbarungen nur schwach mit dem Behandlungserfolg korreliert sind: Zwar treten „Disclosure“-Prozesse bei Patienten mit hoher Belastung vermehrt auf - gleichzeitig profitieren diese aufgrund ihrer hohen Belastung möglicherweise weniger von der Behandlung.

Ein ähnlicher Befund ergibt sich bezüglich der Emotionsverbalisierung, die in den Studien den zweiten, die Katharsis induzierenden Schritt der ,talking cure“ bildet. Die Psychotherapieforschung legt verschiedene Differenzierungen dieses Mechanismus nahe. Erstens werden Emotionen nicht mehr als etwas betrachtet, that needs to be gotten rid of cathartically“ (Greenberg 2012, S. 699), sondern als Erfahrungsaspekt, den es in der Therapie zu be- beziehungsweise verarbeiten gilt. Zweitens ist der Aspekt der Verbalisierung von Emotionen lediglich ein Teilaspekt der ,emotion work": Greenberg (2012) nennt die „expression“ als zweites von sechs emotionsbezogenen ,principles of emotional change“ - hinzu kommen die Prinzipien der „awareness“, „regulation“, „reflection“, „transformation“ und ,corrective emotional experience“ (Greenberg 2012, S. 703 f.). Drittens ist nicht davon auszugehen, dass Emotionsverbalisierungen regelhaft positive Effekte haben. Zwar berichten verschiedene Studien, dass ein höheres Maß des Ausdrucks emotionaler Erfahrungen positiv mit dem Outcome assoziiert ist - andere Studien hingegen zeigen, dass Emotionsverbalisierungen auch mit höherem Stresserleben einhergehen können, beziehungsweise dass die Unterdrückung des Ausdrucks von Emotionen adaptiv sein kann (Greenberg 2012), was insgesamt nahelegt, dass positive Effekte von Emotionsverbalisierungen komplexen Randbedingungen unterliegen, die bislang nur in Teilen verstanden worden sind. 
Zusammenfassend lässt sich festhalten, dass die Psychotherapieforschung zentrale Intuitionen der Katharsislehre der Studien über Hysterie bestätigt, diese aber gleichzeitig modifiziert. Demnach sind kathartische Reinigungsprozesse - meist schlicht als befreiende Entlastungserfahrung gedeutet - als grundlegender therapeutischer Prozess im Sinne eines allgemeinen Wirkfaktors zu betrachten, der sich im Modus verschiedener verbaler Aktivitäten realisieren kann, zum Beispiel dadurch, dass bislang unausgesprochene Erfahrungsinhalte offenbart oder auch Emotionen verbalisiert werden. Wichtig ist allerdings festzuhalten, dass diese verbalen Aktivitäten nicht auf den Katharsiseffekt reduzierbar sind, sondern auch andere Prozesse anstoßen können - so wurden zum Beispiel Selbstoffenbarungsprozesse mit vertieftem Experiencing und Verstehensprozessen assoziiert. Schließlich hat sich gezeigt, dass die kathartischen Effekte der genannten verbalen Aktivitäten komplexen Randbedingungen unterliegen, deren Präzisierung weiterer Forschung bedarf.

\section{Symbolisierung}

Ein zweiter Ansatz beschreibt die ,talking cure“ in symboltheoretischen Begriffen. Symbolisierung wird traditionell als Prozess der Repräsentation eines Y (das Symbolisierte) durch ein X (das Symbol) gefasst (Eco 2015). Als Symbol kommen unterschiedliche Medien in Betracht - Sprache ebenso wie Bilder, Dinge oder Handlungen. Innerhalb der Psychotherapie wurde der Symbolbegriff unterschiedlich verwendet (Lorenzer 1970). Gemeinsam ist den therapeutischen Symboltheorien, das Symbolisierte (Y) auf menschliche Erlebens- und Erfahrungskonstellationen zu beziehen, die durch Symbole beziehungsweise Symbolisierungen ausgedrückt beziehungsweise verändert werden können. Der Fokus der folgenden Ausführungen liegt auf dem Aspekt der Sprachsymbolik, der anhand zweier Modelle bei Gendlin (1997) und Freud (1915) diskutiert wird.

Gendlins Symbolisierungsmodell wurzelt in seiner Theorie des Experiencing, womit ein subjektiver Erfahrungsstrom bezeichnet wird, ,the raw, present, ongoing functioning (in us)“ (Gendlin 1997S. 11). Aufgabe der Therapie sei es, Patienten dabei zu helfen, mit diesem Erfahrungsstrom in Kontakt zu kommen, wobei sie durch die „Focusing“-Methode unterstützt werden können. Bloße Fokussierung reicht aber nicht aus - hinzu kommen muss eine Symbolisierung des Experiencing: „Experiencing is not known, observed, or referred to, except as it is ,symbolized“ in some way“ (Gendlin 1997, S. 238). Gemeint ist damit eine Verknüpfungsoperation - die Verknüpfung eines Erfahrungsaspekts (Experiencing) mit einem Symbol. Dabei ist Gendlins Symbolbegriff breit gefasst: Symbol ist alles, was dazu dienen kann „to express, delineate, explicate, represent, conceptualize ... the felt meaning“ (Gendlin 1997, S. 108). Insgesamt führen Symbolisierungen über den durch Aufmerksamkeitsfokussierung hergestellten Kontakt zum Experiencing hinaus, indem sie subjektive Erfahrungsprozesse in verschiedenerlei Weisen explizieren und hierdurch eine Grundlage für die weitere therapeutische Arbeit schaffen. Diesen Prozess bezeichnet Gendlin auch als „carrying forward“, wobei er von einer fortlaufenden Dialektik von Experiencing- und Symbolisierungsprozessen ausgeht. 
Ein zweites Symbolisierungsmodell lässt sich ausgehend von Freuds Begriffspaar der Sachvorstellung und Wortvorstellung entwickeln. Beide Begriffe bezeichnen Typen mentaler Repräsentationen von Wahrnehmungsphänomenen (Lorenzer 1983). Wortvorstellungen stammen „wesentlich von akustischen Wahrnehmungen“ ab (Freud 1923, S. 283), sind also Repräsentationen gehörter Sprache, die in ihrer Summe den Sprachapparat ausmachen. Den Begriff der Sachvorstellung bezieht Freud auf „Sachbesetzungen der Objekte“ (Freud 1915, S. 160) und lokalisiert diese im Unbewussten. Lorenzer deutet Sachvorstellungen als ,Niederschläge von Lebenspraxis“ (Lorenzer 1983, S. 16), „keine Sach-, sondern Situationsrepräsentanzen, ... Spuren abgelaufener Interaktionen“, ,geronnene Interaktionsformeln“ beziehungsweise Repräsentationen von „Interaktionserfahrungen“, die gleichsam „dynamische Entwürfe, ... virulente Faktoren zukünftiger Lebenspraxis bilden“ (Lorenzer 1983, S. 17). In diesem Sinne präfiguriert der Begriff der Sachvorstellung das Konzept der Selbst- und Objektrepräsentanzen (Blatt et al. 1997).

Die Implikationen des Begriffspaars ergeben sich mit Blick auf die Studie von Freud (1915) über Das Unbewußte, in der es zur Differenzierung bewusster und unbewusster Vorstellungen herangezogen wird: ,[D]ie bewußte Vorstellung umfaßt die Sachvorstellung plus der zugehörigen Wortvorstellung, die unbewußte ist die Sachvorstellung allein“ (Freud 1915, S. 160). Hier geht es also wiederum um eine Verknüpfungsoperation: Sachvorstellungen allein sind unbewusst - erst durch Verknüpfung mit einer Wortvorstellung können sie (vor-)bewusst werden. In den Aphasiestudien (Freud 1891) wurde diese Verknüpfung explizit als symbolische Beziehung gefasst, das heißt, wenn Wort- und Sachvorstellung verknüpft werden, ist die Wortvorstellung (X) die symbolische Repräsentation der Sachvorstellung (Y; Lorenzer 1983). Dies assoziiert Freud (1915) mit der Herbeiführung einer „höhere[n] psychische[n] Organisation“ (S. 160), das heißt der Progression vom Unbewussten zum Vorbewussten beziehungsweise vom Primärprozess zum Sekundärprozess. Diese Lesart hat Implikationen für das Konzept der Verdrängung und die Deutungstechnik: Wenn (Vor-)Bewusstheit von der Verknüpfung von Wort- und Sachvorstellung abhängt, kann als operatives Ziel von Verdrängungsprozessen eben diese Verknüpfung gelten, das heißt, dass Verdrängung entweder verhindert, dass eine Verknüpfung von Wort- und Sachvorstellung zustande kommt, oder aber sie durchtrennt eine bereits bestehende Verknüpfung. Analog lässt sich die Deutungstechnik präzisieren: Wenn Deutungen dazu dienen, unbewusste Phänomene bewusst zu machen, kann dies dadurch erfolgen, dass Verknüpfungen von Wort- und Sachvorstellungen hergestellt beziehungsweise durchtrennte Verknüpfungen wiederhergestellt werden. Vor diesem Hintergrund lässt sich ein globales Modell des psychoanalytischen Prozesses in Termini wort- und sachvorstellungsbezogener Symbolisierungsprozesse skizzieren. In diesem gelten Sachvorstellungen (sensu Selbst- und Objektrepräsentanzen) als Prägungsfaktor menschlichen Erlebens und Verhaltens. Psychopathologie wäre erklärbar als maladaptive Konfiguration von Sachvorstellungen, die jedoch unbewusst (unsymbolisiert) beziehungsweise verdrängt (desymbolisiert) sind - und pathologisch wirksam bleiben. Zentrales Ziel des therapeutischen Prozesses wäre dann, der Unverfügbarkeit pathologisch wirksamer Sachvorstellungen durch deren Bewusstmachung entgegenzuarbeiten, was impliziert, zum Beispiel durch Deutungen Verknüpfungen zwischen spezifischen Sachvorstellungen und adäquaten Wort- 
vorstellungen (wieder-)herzustellen und somit für un- beziehungsweise desymbolisierte Sachvorstellungen eine angemessene Sprache zu finden (Lorenzer 1983).

Im Vergleich der Modelle von Gendlin und Freud zeigen sich zunächst zwei Gemeinsamkeiten: Beide Modelle formulieren globale Konzepte therapeutischer Veränderungsprozesse unter dem Aspekt der Symbolisierung und fassen diese in Termini symbolischer Verknüpfungsoperationen, in deren Vollzug spezifische Erlebens- und Erfahrungsaspekte mit Sprache verknüpft werden. Markant sind aber auch die Unterschiede. Der erste Unterschied betrifft die Erfahrungskonstrukte: Gendlins Experiencing-Begriff ist definiert als globaler Erfahrungsstrom, wohingegen Freuds Erfahrungsbegriff spezifischer als Kristallisation von Interaktionserfahrungen in Form von Selbst- und Objektrepräsentanzen gefasst ist (Lorenzer 1983). Zweitens unterscheiden sich die Modelle hinsichtlich ihrer Annahmen zum Zugriff auf die Erfahrungskonstrukte: Gendlin definiert Experiencing als etwas Bewusstes, Freud hingegen lokalisiert die Sachvorstellungen im Unbewussten, das heißt, ein Zugriff ist ohne Symbolisierung nicht möglich. Der dritte Unterschied besteht hinsichtlich des Symbolbegriffs: Gendlins Modell operiert mit einem breit gefassten Symbolbegriff, der neben Sprachsymbolen auch andere Symboltypen einschließt. Freuds Modell ist demgegenüber - zumindest in seinem ursprünglichen Zuschnitt expliziter auf sprachsymbolische Prozesse fokussiert (Lorenzer 1983).

Empirisch ergibt sich bezüglich beider Modelle ein gemischter Befund. Die in beiden Modellen implizierten Erfahrungskonstrukte können als gut validiert gelten. Gendlins Konzept des Experiencing wird als Maß der Involviertheit des Patienten in den therapeutischen Prozess interpretiert und wurde mit niedrigerer Symptombelastung bei Therapieende assoziiert (Pascual-Leone und Yeryomenko 2017). Ein ähnlicher Befund ergibt sich bezüglich des Konzepts der Sachvorstellung, insofern verschiedene Studien eine höhere Qualität von Objektrepräsentanzen mit einem besseren Behandlungserfolg assoziieren (Blatt et al. 1997). Kaum Evidenz gibt es demgegenüber für die in beiden Modellen implizierten sprachlichen Symbolisierungsprozesse. Zwar gibt es Studien, die eine positive Assoziation zwischen der Focusing-Technik und dem Experiencing-Level (McMullin 1972) beziehungsweise der Inzidenz psychodynamischer Interventionstechniken und dem ,object relations functioning“ (Mullin et al. 2018) nahelegen. Damit sind die in den Modellen postulierten Prozesshypothesen - also dass Symbole mit Sprache verknüpft werden und diese Verknüpfung das Experiencing erhöht beziehungsweise Unbewusstes bewusst macht - allerdings bestenfalls indirekt belegt.

Insgesamt ist festzuhalten, dass Gendlin und Freud theoretische Globalmodelle des therapeutischen Prozesses unter dem Aspekt der Symbolisierung formulieren, die gefasst wird als Verknüpfung von Erlebens- und Erfahrungsaspekten mit Sprache und als heilsam gilt, weil sie unspezifisch verfügbare (Gendlin) beziehungsweise unbewusste (Freud) Erfahrungskonstellationen expliziert beziehungsweise modifiziert. In diesem Sinne bilden beide Modelle einen zentralen Referenzpunkt für das Verständnis von Symbolisierungsprozessen, deren empirische Validität allerdings schwer beurteilbar ist. 


\section{Metaphern}

Der dritte Ansatz beschreibt die „talking cure“ in Termini metaphorischer Sprache. Referenz ist dabei die Poetik des Aristoteles (1997), die Metaphern als Übertragungen eines bestimmten Aspekts $\left(\mathrm{X}_{\mathrm{i}}\right)$ der Bedeutung eines Wortes $(\mathrm{X})$ auf ein anderes Wort (Y) definiert. Über weite Strecken der Rezeptionsgeschichte galten Metaphern als sprachliches Phänomen. Spätestens im 20. Jahrhundert zeichnet sich eine Umdeutung metaphorischer Prozesse ab. Einflussreich war hierbei die Konzeptuelle Metapherntheorie von Lakoff und Johnson (2014), die Metaphorisierung als kognitive Kompetenz mit welterschließender Funktion beschreibt. Kern dieser Kompetenz sei es, eine Entität (Y: „target domain“) in Termini einer anderen Entität (X: ,source domain") zu betrachten, was erreicht werde durch Projekten von Attributen $\left(\mathrm{X}_{\mathrm{i}}\right)$ von X auf Y. Typischerweise ist die ,target domain“ abstrakt, die ,source domain“ hingegen konkret. Deshalb ist Metaphorisierung sinnstiftend: Eine abstraktes Y in Termini eines konkreten $\mathrm{X}$ zu sehen, familiarisiert das $\mathrm{Y}$, macht es plastischer und handhabbarer. Voraussetzung ist allerdings, dass X und Y gemeinsame Attribute aufweisen - ansonsten funktioniert die konzeptuelle Metapher nicht. Dafür ein Beispiel: In Lakoffs klassischer Metapher „love is a journey“ ist ,journey“ die „,source domain“ (X), von der aus Attribute auf die ,target domain“ „love“ (Y) projiziert werden. Dies macht Sinn, weil beide Konzepte gemeinsame Attribute haben (zum Beispiel „Verlauf der Reise“ versus „Verlauf der Beziehung“) - das heißt, die konzeptuelle Metapher eröffnet eine spezifische Perspektive darauf, was „love“ „ist“. Die konzeptuelle Metapher „love is a table“ funktioniert demgegenüber nicht, weil beide Domains keine plausiblen Attribute miteinander teilen. Die Metapher ,love is war" macht wiederum Sinn - eröffnet aber eine andere Perspektive darauf, was „love“ „,ist“. Diese Überlegungen implizieren zweierlei: Erstens, dass Verknüpfungen von ,source“ und ,target“ in Metaphern nicht absolut, sondern variabel sind allerdings nicht beliebig. Zweitens, dass konzeptuelle Metaphern nicht nur Perspektiven eröffnen, sondern gleichzeitig verschließen: Wer seine intimen Beziehungen anhand der ,War“-Metapher strukturiert, wird es schwierig finden, positive Aspekte solcher Beziehungen zu sehen.

In psychotherapeutischen Kontexten wurden die therapeutischen Potenziale metaphorischer Sprache breit diskutiert. Anknüpfungspunkt sind die kommunikativen Möglichkeiten, die Metaphern eröffnen. Fainsilber und Ortony (1987) formulierten hierzu drei Hypothesen: Metaphern können Phänomene beziehungsweise Erlebensweisen ausdrücken, die nicht buchstäblich ausgedrückt werden können (,,inexpressibility hypothesis“) - und dies in verdichteter Form (,compactness hypothesis“) beziehungsweise auf lebendige Weise (,vividness hypothesis“). Klinische Studien unterstreichen diese Hypothesen, indem sie zeigen, dass Metaphern zentrale Themen der Psychotherapie, der Symptomatik des Patienten beziehungsweise von Selbst- und Objektrepräsentanzen zum Ausdruck bringen.

Jenseits der Frage, was Metaphern mitteilen, wurde untersucht, wie in Therapien mit Metaphern gearbeitet wird. Im Vordergrund stehen zwei Prozesse - die Explikation und die Modifikation von Metaphern (Buchholz 2007). Die Explikation dient der Analyse von Metaphern, die von Patienten in die Therapie eingebracht werden, um $\mathrm{zu}$ verstehen, was damit gemeint ist. Dies kann über unterschiedliche Interventionen 
erfolgen, zum Beispiel Wiederholung, Paraphrase oder Klarifikationen. Ein Beispiel für die Explikation einer Metapher geben Bayne und Thompson (2000): Hier formuliert ein Patient die Metapher ,my life is groundhog day“, wobei er Bezug auf den gleichnamigen Film mit Bill Murray nimmt, in dem dieser in einer Zeitschleife gefangen ist und immer wieder denselben Tag durchlebt. Der Therapeut fragt nach: „The same thing every day?" - und setzt damit zu einer Explikation der Metapher an. Die Modifikation von Metaphern kommt bei pathologischen Metaphern zur Anwendung (Buchholz 2007). Das operative Ziel solcher Metaphernarbeit lässt sich in Termini der Konzeptuellen Metapherntheorie präzisieren: Wenn die Verknüpfung von ,source“ und „target“ nicht absolut ist, kann ein ,target“" seine Projektionen von verschiedenen ,sources“ empfangen. Damit eröffnet sich die Perspektive, in Therapien im Fall maladaptiver Quelle-Ziel-Verknüpfungen auf eine Veränderung dieser Verknüpfung hinzuarbeiten, das heißt, jeweils ,target domains“ mit neuen (adaptiveren) „source domains“ zu assoziieren. Ein Beispiel für diese Praxis bieten Levitt et al. (2000), die zeigen, wie im Verlauf der Therapie einer Patientin mit Depressionen die Frequenz der belastungsassoziierten (,burden“) Metaphern (zum Beispiel „my heart feels heavy“) abnahm, wohingegen die Zahl der entlastungsassoziierten (,unburdening“) Metaphern (zum Beispiel ,the pressure has lifted“) zunahm.

Bezüglich der Effekte therapeutischer Metaphernarbeit ist die Befundlage in vielen Hinsichten unklar. Einige Studien berichten positive Assoziationen zwischen Metapherngebrauch und Prozessvariablen. So zeigte sich zum Beispiel, dass Patienten Redephasen von Therapeuten mit (vs. ohne) Metaphern beziehungsweise Sitzungen mit (vs. ohne) intentionalem Metapherngebrauch durch Therapeuten als hilfreicher bewerten (McMullen 2008). Insbesondere scheinen Metaphern Problemlösungsprozesse (,,problem-setting and -solving“) zu erleichtern und Verstehensprozesse (,,insight“") zu begünstigen (McMullen 2008). Viele Detailfragen der Metaphernarbeit sind aber umstritten. Kontrovers diskutiert wurde die Frage, ob unkonventionelle (,novel“) Metaphern in der Therapie hilfreicher sind als konventionelle (,frozen“) Metaphern: Einige Studien erbrachten Evidenz für diese Position, andere hingegen nicht (McMullen 2008). Wichtiger als die Originalität der Metapher scheint zu sein, ob Metaphern für den Patienten signifikant sind - und dies können durchaus auch konventionelle Metaphern sein, die Kernthemen eines Patienten beziehungsweise der Therapie zum Ausdruck bringen (McMullen 2008). Umstritten ist auch, inwiefern Metapherngebrauch mit Experiencing-Prozessen assoziiert ist: Einzelne Studien assoziierten metaphorische Sprache mit erhöhten Experiencing-Leveln (Gelo und Mergenthaler 2012), andere Studien fanden einen negativen Zusammenhang (Stuart 1997). Zur Deutung der negativen Assoziationen wurde vorgeschlagen, dass Metaphern auch zu Abwehrzwecken gebraucht werden können, was nahelegt, dass Metapherngebrauch nicht notwendigerweise mit einem produktiven therapeutischen Prozess assoziiert sein muss.

Insgesamt zeigen sich in der Forschung Evidenzen für den produktiven Effekt therapeutischer Metaphernarbeit. Im Vordergrund steht dabei zunächst das kommunikative Potenzial von Metaphern, komplexe Erfahrungskonstellationen kompakt und lebendig zu versprachlichen, was vor allem mit Verstehens- und Problemlösungsprozessen assoziiert wurde. Weiterhin eröffnet Metaphernarbeit Perspektiven für Veränderungsprozesse, etwa die Korrektur maladaptiver Selbst- oder Krankheits- 
konzepte, die sich in Metaphern ausdrücken. Festzuhalten ist schließlich der Befund, dass Metaphern auch der Abwehr dienen können, was zeigt, dass das produktive Potenzial der Metaphernarbeit spezifischen Randbedingungen unterliegt, die weiter zu erforschen sind.

\section{Verbale Interventionen}

Der vierte Ansatz beschreibt die ,talking cure“ in Termini verbaler Interventionen. In den Fokus rücken damit sprachliche Techniken, die in Therapien als strategisch einsetzbare Werkzeuge dienen, um bestimmte Effekte zu erzielen. Die meisten Therapieschulen definieren solche Techniken - die Psychoanalyse etwa Deutung, Konfrontation und Klarifikation, die Verhaltenstherapie positive Selbstverbalisierung oder ,prompting“, die Systemische Therapie das zirkuläre Fragen oder die Klientenzentrierte Gesprächspsychotherapie das „focusing“ (Gumz et al. 2017). Diese verbalen Techniken synoptisch zu integrieren, ist schwierig: Zwar liegen theoretische Systematisierungsversuche vor, diese zeigen allerdings erhebliche definitorische Unschärfen (Gumz et al. 2014), was eine präzise Operationalisierung verbaler Techniken erschwert. Folglich existiert in der Forschung eine Vielzahl disparater Erhebungsinstrumente, die identische Konstrukte unterschiedlich definieren, verschiedene Aspekte verbaler Techniken akzentuieren und psychometrischen Gütekriterien zum Teil unzureichend gerecht werden (Gumz et al. 2015). Dies trägt zur Heterogenität der Befundlage bei, was eine Evaluation der Effektivität verbaler Techniken verkompliziert. Dies sei im Folgenden exemplarisch anhand der Technik der Übertragungsdeutung illustriert.

Die Übertragungsdeutung gilt als eine zentrale Technik der Psychoanalyse. Grundsätzlich ist sie ein Spezialfall der Deutung, das heißt die Anwendung der Deutungstechnik (formaler Aspekt) auf Übertragungsphänomene (inhaltlicher Aspekt; Gumz et al. 2014). In der Forschung zeichnet sich die Tendenz ab, Überragungsdeutungen nicht mehr als einheitlichen Interventionstyp zu fassen, sondern als Sammelbegriff für verschiedene übertragungsbezogene Interventionen. Oft ist von „Übertragungsarbeit“ (,transference work“) die Rede, die Høglend (2014) definiert als ,,any therapist intervention that points out, refers to, wonders about, or explains the patient's experience of the therapist and the ongoing patient-therapist interaction“ (Høglend 2014, S. 1057). Die Befundlage zu Effekten von Übertragungsarbeit ist komplex. Verschiedene Studien berichten, dass höhere Frequenzen von Übertragungsdeutungen mit besserem Outcome einhergehen, wohingegen andere Studien negative Outcome-Assoziationen fanden (Brumberg und Gumz 2012). Dies legt nahe, dass der Zusammenhang von Übertragungsarbeit und Outcome von Drittvariablen beeinflusst wird. Im Zentrum der Diskussion stehen der moderierende Effekt der Objektbeziehungsqualität sowie der therapeutischen Allianz: Bezüglich der Objektbeziehungsqualität deuten Befunde darauf hin, dass Patienten mit niedriger Qualität der Objektbeziehungen beziehungsweise damit einhergehendem höheren $\mathrm{Maß}$ an interpersonellen Problemen mehr von Übertragungsarbeit profitieren als Patienten mit höherer Objektbeziehungsqualität (Høglend 2014). Dies unterstreicht die Position der übertragungsfokussierten Psychotherapie, strukturschwachen Pati- 
enten mit Übertragungsdeutungen helfen zu können. Bezüglich der therapeutischen Allianz wurde diskutiert, ob diese durch einen Fokus auf Übertragungsarbeit beeinträchtigt werden kann, etwa weil Patienten Übertragungsdeutungen als konfrontativ erleben. Diesbezüglich ergab sich der differenzierende Befund, dass die Effekte von Übertragungsdeutungen auf die Allianz von verschiedenen Bedingungen abhängen, etwa der Objektbeziehungsqualität des Patienten oder auch dem Status der Allianz vor Gabe der Übertragungsdeutung (Brumberg und Gumz 2012). Weiterhin werden die Effekte von Übertragungsarbeit von verschiedenen anderen Variablen beeinflusst, zum Beispiel Charakteristika des Patienten (zum Beispiel Behandlungsmotivation, Symptomatik, Lebensqualität, Geschlecht), des Therapeuten (zum Beispiel Gegenübertragung) sowie der Übertragungsdeutung selbst (zum Beispiel Exaktheit der Deutung; Brumberg und Gumz 2012). Insgesamt ist die Befundlage also kompliziert, was die Formulierung evidenzbasierter Praxisempfehlungen erschwert. Deutlich wird immerhin: Übertragungsdeutungen sind nicht an und für sich nützlich oder schädlich - ihr Effekt hängt von spezifischen Randbedingungen ab. Dies impliziert, dass Übertragungsarbeit nicht aus Prinzip, sondern mit Umsicht, in Maßen und gezielt sowie mit Blick auf den Stand der Therapie sowie auf die spezifischen Umstände von Patient und Therapeut betrieben werden sollte (Brumberg und Gumz 2012).

Was am Beispiel der Übertragungsdeutung gezeigt wurde, kann als repräsentativ für die allgemeine Befundlage zu Effekten verbaler Interventionstechniken gelten. Regelhaft sind die empirischen Evidenzen schwer interpretierbar - und folglich lässt sich kaum ein Beispiel für eine Technik aufweisen, die in exakt definierter Form für alle Patienten typischerweise positive Effekte aufweist (Barber et al. 2013). Gleichwohl resultiert aus der assoziierten Forschung ein wichtiger Befund: Bezüglich verbaler Techniken ist grundsätzlich kein linearer Haupteffekt zu erwarten - vielmehr hängt ihre Wirksamkeit von einem komplexen Gefüge weiterer - zum Teil nichtsprachlicher - Faktoren ab, die näher zu bestimmen Aufgabe weiterer Forschung sein wird.

\section{Narrative}

Der fünfte Ansatz beschreibt die „talking cure“ in narrativen Begriffen. Zu differenzieren sind dabei zwei Bezugsebenen: Auf der ersten Ebene bezieht sich der Begriff des Narrativs auf die Konstruktion individueller Selbstkonzepte, das heißt, das Selbst wird als Narrativ betrachtet und die Herausbildung des Selbst als narrativer Prozess (Bruner 1990). Auf der zweiten Ebene bezieht sich der Begriff auf die lokale Ebene kommunikativer Prozesse. Ausgangspunkt ist hier die anthropologische These vom Menschen als „homo fabulans“, welche die Fähigkeit zur Narrativierung als evolutionär entwickelte kognitive Kompetenz interpretiert (Bruner 1990). In diesem Sinne ist das Erzählen von Geschichten ein basaler Modus der Formatierung verbaler Interaktion, dessen Funktion darin besteht, Erfahrungsinhalte als linear-chronologisches Narrativ zu strukturieren.

Im Rahmen der Psychotherapieforschung lieferte die Narrationstheorie verschiedene Perspektiven für die therapeutische Praxis. Angeknüpft wird dabei zunächst am 
Aspekt des Selbstnarrativs, der eine Beschreibung von Psychopathologie im Sinne maladaptiver narrativer Selbstkonstruktionsprozesse nahelegt. In diesem Sinne lassen sich für verschiedene Störungsbilder spezifische Aspekte narrativer Selbstkonstruktion nachweisen: Selbstnarrative depressiver Patienten beinhalten zum Beispiel inhaltlich mehr negative Ereignisse, sind stärker von einem negativen Attributionsstil geprägt und weichen stärker von der linearen chronologischen Ordnung ab als die Narrative einer nichtdepressiven Vergleichsgruppe (Habermas et al. 2008). Diese Befunde implizieren, auch therapeutische Praxis unter dem Aspekt narrativer Prozesse zu betrachten: Wenn psychische Störungen als maladaptive Selbstnarrative interpretierbar sind, wird Psychotherapie zum Prozess der Veränderung solcher Narrative.

Beforscht wurden weiterhin die Effekte narrativer Prozesse in der Psychotherapie. Verschiedene Studien berichten, dass Patienten das Erzählen eigener Erfahrungen in der Therapie als kathartisch empfinden, insofern es eine Möglichkeit biete, mit schwierigen Themen in Kontakt zu treten und diese zu verarbeiten (Rennie 1994). Weiterhin wurde das Erzählen der eigenen Geschichte als Möglichkeit beschrieben, zu einem differenzierteren Verstehen bestimmter Erfahrungen zu gelangen beziehungsweise die eigene Biografie insgesamt neu zu fassen (Stiles et al. 1999). Gezeigt wurde aber auch, dass narrative Prozesse im Dienst der Abwehr stehen können: So berichten Patienten, dass sie das Geschichtenerzählen als Strategie benutzen, um sich von belastenden Erfahrungen zu distanzieren (Gonçalves et al. 2017; Rennie 1994), was nahelegt, dass narrative Prozesse nicht notwendigerweise mit einem produktiven Behandlungsverlauf assoziiert sein müssen.

Weitere Befunde ergeben sich aus der Forschung zu narrativen Prozessen auf Grundlage quantitativer Rating-Systeme. Einflussreich ist in diesem Zusammenhang zunächst das „Narrative-emotion-process(N-EP)“-Modell (Angus et al. 2017), das in seiner ersten Fassung drei Typen narrativer Sequenzen unterscheidet: Externale Sequenzen, in denen Patienten äußere Ereignisse beschreiben, internale Sequenzen, in denen innere Prozesse (zum Beispiel Gefühle) beschrieben werden, und reflexive Sequenzen, in denen Patienten darüber reflektieren, was bestimmte Ereignisse für sie bedeuten. Studien auf Grundlage des N-EP-Modells zeigen, dass Narrative von Patienten über die Therapie hinweg spezifischer werden (Angus 2012). Zudem ergaben sich Unterschiede im narrativen Stil bei Patienten mit positivem vs. negativem Outcome: Patienten mit positivem Outcome zeigen mehr reflexive Erzählsequenzen, einen Anstieg der Frequenz reflexiver Sequenzen über die Therapie hinweg, einen höheren Anteil von Wechseln zwischen internalen und reflexiven Sequenzen sowie eine stärkere Neigung zum Emotionsausdruck und höheres emotionales Arousal beim Erzählen autobiografischer Erinnerungen (Angus 2012). In der zweiten Fassung des N-EP wurde die Typologie narrativer Sequenzen durch eine Typologie narrativer Emotionsmarker ersetzt: Problemmarker, die unter- sowie überregulierte oder auch undifferenzierte narrative Prozesse beschreiben (zum Beispiel ,empty storytelling“: inhaltlich und emotional leere Geschichte), Übergangsmarker, die durch Prozesse vermehrter Reflexivität und vermehrtem Emotionsausdruck gekennzeichnet sind (zum Beispiel ,competing plotlines storytelling“: Auftauchen einer Alternative zur alten, maladaptiven Geschichte), und Veränderungsmarker, die mit produktiven narrativ-emotionalen Integrationsprozessen assoziiert sind (zum Beispiel „discovery 
storytelling“: Rekonzeptualisierung des Selbstverständnisses; (Angus et al. 2017). Studien auf Grundlage des revidierten Systems zeigen, dass Patienten mit positivem Outcome in ihren narrativen Prozessen weniger Problem- und mehr Veränderungsmarker zeigen und eine stärkere Neigung zum Wechsel von Problem- zu Veränderungsmarkern aufweisen, was ein höheres Maß an narrativer Flexibilität impliziert (Angus et al. 2017).

Einen alternativen Ansatz spezifiziert das „Innovative-moments“-Modell (Gonçalves et al. 2017). Kern ist auch hier eine Systematik narrativer Prozesse, die unter dem Aspekt ,innovativer Momente“ (IM) entfaltet wird. Bezeichnet werden damit Anomalien in narrativen Prozessen, etwa wenn eine selbstunsichere Patientin in der Therapie davon erzählt, wie sie in einer schwierigen Situation eigene Bedürfnisse durchsetzt. In solchen Anomalien zeigen sich Alternativen zu ansonsten dominanten maladaptiven Erlebens- und Verhaltensmustern, die es in der Therapie aufzugreifen und zu stärken gilt. Unterschieden werden sieben Typen innovativer Momente, die zwei Basistypen zugeordnet werden: „low-level IM“", die eine Distanzierung von der Problematik indizieren (zum Beispiel ,reflection 1“: neues Verständnis eines Problems), und „high-level IM“, die Veränderungsprozesse anzeigen (zum Beispiel ,reconceptualization“: metareflexive Wahrnehmung von Veränderungsprozessen). Befunde assoziierter Studien zeigen, dass die Zahl der IM bei Patienten mit positivem Outcome höher ist als bei Patienten mit schlechtem Outcome, dass eine höhere Zahl der IM Symptomverbesserungen in der folgenden Sitzung vorhersagt, und dass High-level IM für die Initiierung therapeutischer Veränderungsprozesse bedeutsamer sind als Low-level IM (Gonçalves et al. 2017). Ein zweiter Strang IM-assoziierter Forschung untersucht die Effekte nichtgenutzter innovativer Momente. Zur Untersuchung solcher Prozesse wurde das Konzept des „Return-to-the-problem“(RPM)Markers entwickelt, der als Indikator für Therapieambivalenz gilt (Gonçalves et al. 2017). Verschiedene Studien belegen, dass Patienten mit gutem Outcome weniger RPM als Patienten mit Patienten mit schlechtem Outcome zeigen, dass die Frequenz von RPM-Markern im Zuge von Symptomverbesserungen sinkt, und dass eine Reduktion von RPM eine Symptomverbesserung in der folgenden Stunde vorhersagt (Gonçalves et al. 2017).

Insgesamt offeriert die narrative Forschung eine spezifische Perspektive auf die „talking cure“ in Termini des Geschichtenerzählens. Daraus ergibt sich einerseits eine übergreifende Perspektive, die die Selbstkonstruktion als narrativen Prozess, Psychopathologie als Resultat maladaptiver Selbstkonstruktion und Psychotherapie als Veränderung pathologischer Selbstnarrative interpretiert. Andererseits arbeitet die Forschung zu narrativen Prozessen auf lokaler Ebene die komplexen Modalitäten der Expression, Reflexion und Integration problematischer Erfahrungen im Medium narrativer Prozesse sowie deren Beitrag zu günstigen Behandlungsverläufen heraus - akzentuiert dabei allerdings auch den Umstand, dass Geschichtenerzählen nicht an und für sich produktiv sein muss, insofern es auch im Dienst defensiver Prozesse stehen kann. 


\section{Resümee}

In der Zusammenschau erweist sich die ,talking cure“ als ebenso komplexes wie heterogenes Phänomen. Heterogen ist zum einen die theoretische Konzeptualisierung der therapeutischen Wirkung von Sprache: Die „talking cure“ wird theoretisch in Termini disparater Ansätze ausbuchstabiert, die ein breites Spektrum von - teils spezifisch (zum Beispiel Emotionsverbalisierungen), teils breiter (zum Beispiel Narrative) gefassten - verbalen Aktivitäten in den Blick nehmen, den therapeutischen Effekt dieser Aktivitäten in Relation zu einem breiten Spektrum von - wiederum teils spezifisch (zum Beispiel Emotionen), teils breiter (zum Beispiel Experiencing) gefassten - Erfahrungskonstrukten beschreiben und in diesem Zusammenhang unterschiedliche Veränderungsmechanismen postulieren. Im Überblick lässt sich als gemeinsamer Nenner der verschiedenen Ansätze die Annahme festhalten, dass Sprache deshalb therapeutisch wirksam sein kann, weil sie in der Therapie eine zentrale Möglichkeit zur Explikation beziehungsweise Modifikation pathologisch-maladaptiver Erlebens- und Erfahrungskonstellationen bietet. Dies allerdings kann Unterschiedliches bedeuten: Die ,talking cure“ kann darin bestehen, dass Patienten durch Emotionsverbalisierungen oder Selbstoffenbarungen kathartische Erleichterung erfahren; dass im Zuge der Therapie Symbolisierungen unklarer oder unbewusster Erlebens- und Erfahrungskonstellationen erarbeitet werden; dass eine Metapher geprägt wird, die in verdichteter Form eine Erlebens- oder Erfahrungskonstellation zum Ausdruck bringt, dass eine Therapeutin dem Patienten durch eine Deutung ein Übertragungsmuster verfügbar macht, oder dass Patientin und Therapeut im Zuge eines narrativen Prozesses ein negatives Selbstnarrativ korrigieren - und all diese und weitere Prozesse können parallel ablaufen, sich ergänzen und überschneiden.

Heterogen ist zum anderen die empirische Befundlage bezüglich der ,talking cure". Gleichwohl lassen sich drei Tendenzen der Forschung ausmachen:

Erstens ist die Assoziation verschiedener Typen verbaler Aktivität mit dem Outcome (das heißt dem Behandlungserfolg) regelhaft unklar: Oft wurden zwischen verbalen Aktivitäten und Outcome keine oder nur schwache Assoziationen gefunden (zum Beispiel bei Selbstoffenbarungsprozessen), oder aber die Assoziationen sind widersprüchlich (zum Beispiel bei Übertragungsdeutungen). Insgesamt legt dieses Befundmuster nahe, bezüglich der meisten verbalen Aktivitäten keinen Haupteffekt auf das Outcome anzunehmen - was wiederum ein simplifizierendes technisches Verständnis sprachlicher Vollzüge, demzufolge spezifische Typen verbaler Aktivität (X) regelhaft spezifische Effekte (Y) haben (Stiles 2017), differenziert. Auszugehen ist vielmehr vom Regelfall komplexer Zusammenhänge zwischen verbaler Aktivität und Outcome, womit sich der Analysefokus auf die Randbedingungen positiver Effekte verbaler Aktivität und mithin die moderierende und mediierende Funktion von Drittvariablen verschiebt.

Konsistentere Befunde finden sich - zweitens - bei Assoziationen verbaler Aktivitäten mit Prozessvariablen. Auffallend ist, dass dieselben Prozessvariablen oft mit unterschiedlichen verbalen Aktivitäten in Verbindung gebracht wurden - kathartische Erleichterung zum Beispiel mit Selbstoffenbarungen, Emotionsverbalisierungen und Narrativen; Verstehensprozesse mit Übertragungsdeutungen, Metaphern und Narrativen, Experiencing mit Selbstoffenbarungen, Metaphern und Narrativen 
oder auch eine positive therapeutische Beziehung mit Selbstoffenbarungen, Emotionsverbalisierungen, Metaphern und Narrativen. Dieses Befundmuster impliziert, dass bestimmte veränderungsrelevante Prozesse über unterschiedliche Typen verbaler Aktivität realisiert werden können, was die Notwendigkeit einer integrativen Perspektive auf den Beitrag sprachlicher Aktivität im Kontext therapeutischer Veränderungsprozesse unterstreicht.

Drittens ist zu bedenken, dass bezüglich der meisten verbalen Aktivitäten auch negative Effekte nachgewiesen wurden: Selbstoffenbarungen und Emotionsverbalisierungen können mit erhöhtem Stress einhergehen, ungünstig platzierte Übertragungsdeutungen die therapeutische Allianz beschädigen, Metaphern und Narrative im Dienst von Abwehrprozessen stehen. Damit wird deutlich, dass Sprache in der Therapie ein zweischneidiges Schwert ist, das nicht nur positive Prozesse in Gang bringt, sondern auch Schaden anrichten kann.

Insgesamt zeigt sich mit Blick auf die Psychotherapieforschung, dass es keine einfache Antwort auf die Frage gibt, wie „Reden“ in der Therapie „heilen“ kann. Grundsätzlich legt die Empirie nahe, heilsame Effekte verbaler Aktivität in der Psychotherapie als Teilaspekt eines komplexen Gefüges multipler miteinander interagierender Faktoren zu betrachten, die nur in Teilen manifest und grundsätzlich im Kontext eines komplexen, von nonverbalen (Benecke et al. 2005) beziehungsweise vor- und unbewussten (Lorenzer 2002) Aspekten maßgeblich geprägten Beziehungsgeschehens zu betrachten und analysieren sind. Weiterhin ist anzunehmen, dass Sprache - innerhalb dieses Beziehungsgeschehens - über ein Set basaler Prozesse operiert (zum Beispiel kathartische Erleichterung, Verstehen, Experiencing), die über unterschiedliche Typen verbaler Aktivität induziert werden können - und die $\mathrm{zu}$ systematisieren und in einem integrativen Modell zusammenzufassen ein wichtiges Ziel der weiteren Forschung darstellt. Wichtig erscheint es diesbezüglich, zunächst den theoretischen Zugriff auf die ,talking cure“ zu präzisieren und insbesondere die Aspekte der verbalen Aktivität, der hierdurch adressierten Erlebens- und Erfahrungskonstellationen sowie der beteiligten veränderungsassoziierten Prozesse zu differenzieren (Marx et al. 2017). Auf dieser Grundlage erst können adäquate Definitionen und Operationalisierungen relevanter Konstrukte formuliert werden (Gumz et al. 2015), die wiederum einen fokussierten, auf quantitative (Gumz et al. 2014) und auch qualitative (Buchholz und Kächele 2017; Georgaca und Avdi 2009) Methoden gestützten Forschungsprozess ermöglichen, um somit letztlich zu einem konkreteren Verständnis des spezifischen Beitrags der Sprache im Kontext therapeutischer Veränderungsprozesse zu gelangen.

Funding Open Access funding provided by Projekt DEAL.

Open Access Dieser Artikel wird unter der Creative Commons Namensnennung 4.0 International Lizenz veröffentlicht, welche die Nutzung, Vervielfältigung, Bearbeitung, Verbreitung und Wiedergabe in jeglichem Medium und Format erlaubt, sofern Sie den/die ursprünglichen Autor(en) und die Quelle ordnungsgemäß nennen, einen Link zur Creative Commons Lizenz beifügen und angeben, ob Änderungen vorgenommen wurden.

Die in diesem Artikel enthaltenen Bilder und sonstiges Drittmaterial unterliegen ebenfalls der genannten Creative Commons Lizenz, sofern sich aus der Abbildungslegende nichts anderes ergibt. Sofern das betreffende Material nicht unter der genannten Creative Commons Lizenz steht und die betreffende Handlung 
nicht nach gesetzlichen Vorschriften erlaubt ist, ist für die oben aufgeführten Weiterverwendungen des Materials die Einwilligung des jeweiligen Rechteinhabers einzuholen.

Weitere Details zur Lizenz entnehmen Sie bitte der Lizenzinformation auf http://creativecommons.org/ licenses/by/4.0/deed.de.

Interessenkonflikt C. Marx, C. Benecke und A. Gumz geben an, dass kein Interessenkonflikt besteht.

\section{Literatur}

Angus LE (2012) Toward an integrative understanding of narrative and emotion processes in emotion-focused therapy of depression: implications for theory, research and practice. Psychother Res 22:367-380

Angus LE, Boritz T, Bryntwick E, Carpenter N, Macaulay C, Khattra J (2017) The narrative-emotion process coding system 2.0: a multi-methodological approach to identifying and assessing narrativeemotion process markers in psychotherapy. Psychother Res 27:253-269

Aristoteles (1997) Poetik. Reclam, Stuttgart

Barber JP, Muran JC, McCarthy KS, Keefe RJ (2013) Research on psychodynamic therapies. In: Lambert MJ (Hrsg) Bergin and Garfield's handbook of psychotherapy and behavior change, 6. Aufl. John Wiley \& Sons Inc., Hoboken, S 443-494

Bayne R, Thompson KL (2000) Counsellor response to clients' metaphors: An evaluation and refinement of Strong's model. Couns Psychol Q 13:37-49

Benecke C, Peham D, Bänninger-Huber E (2005) Nonverbal relationship regulation in psychotherapy. Psychother Res 15:81-90

Blatt SJ, Auerbach JS, Levy KN (1997) Mental representations in personality development, psychopathology, and the therapeutic process. Rev Gen Psychol 1:351-374

Breuer J, Freud S (1895) Studien über Hysterie. Fischer, Frankfurt/Main (2007)

Brumberg J, Gumz A (2012) Was sind Übertragungsdeutungen und wie wirken sie? Eine systematische Übersicht. Z Psychosom Med Psychother 58:219-235

Bruner J (1990) Acts of meaning. Harvard University Press, Cambridge und London

Buchholz MB (2007) Listening to words, seeing images: Metaphors of emotional movement. Int Forum Psychoanal 16:164-176

Buchholz MB, Kächele H (2017) From turn-by-turn to larger chunks of talk: An exploratory study in psychotherapeutic micro-processes using conversation analysis. Res Psychother Psychopathol Process Outcome 20:161-178

Eco U (2015) Zeichen: Einführung in einen Begriff und seine Geschichte. Suhrkamp, Frankfurt a.M. (1977)

Fainsilber L, Ortony A (1987) Metaphorical uses of language in the expression of emotions. Metaphor Symb Activity 2:239-250

Farber BA (2003) Patient self-disclosure: a review of the research. J Clin Psychol 59:589-600

Freud S (1891) Zur Auffassung der Aphasien. Fischer, Frankfurt a.M. (2001)

Freud S (1915) Das Unbewußte. In: Freud S (Hrsg) Psychologie des Unbewußten: Studienausgabe, Bd. III. Fischer, Frankfurt. a.M., S 119-173 (2000)

Freud S (1916) Vorlesungen zur Einführung in die Psychoanalyse und Neue Folge: Studienausgabe Bd. I. Fischer, Frankfurt a.M. (1916-17)

Freud S (1923) Das Ich und das Es. In: Freud S (Hrsg) SA III, S 273-330

Gelo OCG, Mergenthaler E (2012) Unconventional metaphors and emotional-cognitive regulation in a metacognitive interpersonal therapy. Psychother Res 22:159-175

Gendlin ET (1997) Experiencing and the creation of meaning. Northwestern University Press, Evanston

Georgaca E, Avdi E (2009) Evaluating the talking cure: The contribution of narrative, discourse, and conversation analysis to psychotherapy assessment. Qual Res Psychol 6:233-247

Gonçalves MM, Ribeiro AP, Mendes I, Alves D, Silva J, Rosa C, Braga C, Batista J, Fernández-Navarro P, Oliveira JT (2017) Three narrative-based coding systems: Innovative moments, ambivalence and ambivalence resolution. Psychother Res 27:270-282

Greenberg LS (2012) Emotions, the great captains of our lives: Their role in the process of change in psychotherapy. Am Psychol 67:697-707

Gumz A, Horstkotte JK, Kästner D (2014) Das Werkzeug des psychodynamischen Psychotherapeuten Verbale Interventionstypen aus theoretischer und aus der Praxis abgeleiteter Perspektive. Z Psychosom Med Psychother 60:219-237 
Gumz A, Neubauer K, Horstkotte JK, Geyer M, Löwe B, Murray AM, Kästner D (2017) A bottom-up approach to assess verbal therapeutic techniques. Development of the Psychodynamic Interventions List (PIL). PLoS ONE. https://doi.org/10.1371/journal.pone.0182949

Gumz A, Treese B, Marx C, Strauss B, Wendt H (2015) Measuring verbal psychotherapeutic techniques-A systematic review of intervention characteristics and measures. Front Psychol. https://doi. org/10.3389/fpsyg.2015.01705

Habermas T, Ott L-M, Schubert M, Schneider B, Pate A (2008) Stuck in the past: Negative bias, explanatory style, temporal order, and evaluative perspectives in life narratives of clinically depressed individuals. Depress Anxiety 25:E121-E132

Høglend P (2014) Exploration of the patient-therapist relationship in psychotherapy. Am J Psychiatry 171:1056-1066

Kelly AE (1998) Clients' secret keeping in outpatient therapy. J Couns Psychol 45:50-57

Lakoff G, Johnson M (2014) Leben in Metaphern. Konstruktion und Gebrauch von Sprachbildern. Carl Auer, Heidelberg

Lorenzer A (1970) Kritik des psychoanalytischen Symbolbegriffs. Suhrkamp, Frankfurt a.M.

Lorenzer A (1983) Sprache, Lebenspraxis und szenisches Verstehen in der psychoanalytischen Therapie. Psyche (Stuttg) 37:97-115

Lorenzer A (2002) Die Sprache, der Sinn, das Unbewußte. Klett-Cotta, Stuttgart

Marx C, Benecke C, Gumz A (2017) Talking cure models: A framework of analysis. Front Psychol. https:// doi.org/10.3389/fpsyg.2017.01589

McMullen LM (2008) Putting it in context: metaphor and psychotherapy. In: Gibbs RW (Hrsg) The Cambridge handbook of metaphor and thought. Cambridge University Press, New York, S 397-411

McMullin RE (1972) Effects of conselor focusing on client self-experiencing under low attitudinal conditions. J Couns Psychol 19:282-285

Mullin ASJ, Hilsenroth MJ, Gold J, Farber BJ (2018) Facets of object representation: process and outcome over the course of psychodynamic psychotherapy. J Pers Assess 100:145-155

Pascual-Leone A, Yeryomenko N (2017) The client "experiencing" scale as a predictor of treatment outcomes: a meta-analysis on psychotherapy process. Psychother Res 27:653-665

Rennie DL (1994) Storytelling in psychotherapy: the client's subjective experience. Psychother Theory Res Pract Train 31:234-243

Stiles WB (1995) Disclosure as a speech act: Is it psychotherapeutic to disclose? In: Pennebaker JW (Hrsg) Emotion, disclosure, \& health. American Psychological Association, Washington, S 71-91

Stiles WB (2017) Finding the right words: symbolizing experience in practice and theory. Pers Cent Exp Psychother 16:1-13

Stiles WB, Honos-Webb L, Lani JA (1999) Some functions of narrative in the assimilation of problematic experiences. J Clin Psychol 55:1213-1226

Stuart JJ (1997) Novel figurative language and patient experiencing in psychodynamic therapy. Psychother Res 7:219-237

Tschacher W, Junghan UM, Pfammatter M (2014) Towards a taxonomy of common factors in psychotherapy-Results of an expert study. Clin Psychol Psychother 21:82-96

Wampold BE (2007) Psychotherapy: the humanistic (and effective) treatment. Am Psychol 62:855-873

Woolfolk RL (2015) The value of psychotherapy: The talking cure in an age of clinical science. Guilford, New York, London

Dipl.-Psych. Christopher Marx Jg. 1978, psychologischer Psychotherapeut in eigener Praxis, Dozent an der Psychologischen Hochschule Berlin, der Berliner Akademie für Psychotherapie und der Kunsthochschule Berlin-Weißensee. Arbeitsschwerpunkte: Sprache in der Psychotherapie, psychoanalytische Konzeptforschung, symbolische Heilpraktiken, therapeutische Allianz.

Prof. Dr. phil. Dipl.-Psych. Cord Benecke psychologischer Psychotherapeut, Psychoanalytiker, Jg. 1965; Professor für klinische Psychologie und Psychotherapie, Institut für Psychologie der Universität Kassel; Forschungsschwerpunkte: klinische Emotionsforschung, Psychotherapieforschung.

Prof. Dr. med. Antje Gumz Fachärztin für psychosomatische Medizin und Psychotherapie (TP), Psychoanalytikerin, systemische Therapeutin (DGSF); Professorin für Psychosomatik und Psychotherapie, Psychologische Hochschule Berlin; Forschungsschwerpunkte: Psychotherapie- und Ausbildungsforschung (Spannungen und Krisen in der Therapiebeziehung, Messen interpersoneller Fähigkeiten von Therapeuten, Sprache in der Psychotherapie, psychodynamische Interventionstechniken); Burn-out und psychogene Arbeitsstörungen; Prävention der Anorexia nervosa. 